\title{
Comparison of HREM images and contrast simulations for dodecagonal $\mathrm{Ni}-\mathrm{Cr}$ quasicrystals
}

\author{
C. Beeli $\left({ }^{1}\right)$, F. Gähler $\left({ }^{2}\right)$, H.-U. Nissen $\left({ }^{1}\right)$ and P. Stadelmann $\left({ }^{3}\right)$ \\ (1) Laboratorium für Festkörperphysik, ETH-Hönggerberg, CH-8093 Zürich, Switzerland \\ (2) Dépt de Physique Théorique, Université de Genève, 24 quai Ernest Ansermet, \\ $\mathrm{CH}-1211$ Genève 4, Switzerland \\ $\left.{ }^{3}\right)$ Inst. Interdépartemental de Microscopie Electronique, $\mathbf{I}^{2} \mathbf{M}$, Ecole Polytechnique Fédérale \\ Lausanne, CH-1015 Lausanne, Switzerland
}

(Reçu le 11 octobre 1989, accepté sous forme définitive le 7 décembre 1989)

\begin{abstract}
Résumé. - Du fait de la similarité de contraste entre micrographies électroniques haute résolution des quasi-cristaux dodécagonaux $\mathrm{Ni}-\mathrm{Cr}$ et celles de plusieurs phases périodiques voisines, il a été suggéré que les quasi-cristaux dodécagonaux peuvent être décrits comme une décoration d'un pavage quasi-périodique dodécagonal renfermant les mêmes unités structurales que celles qui interviennent dans ces phases périodiques. Dans le but de corroborer cette hypothèse, des simulations de contraste en microscopie électronique utilisant de tels modèles structuraux sont présentées. Les modèles considérés sont basés sur différents pavages quasipériodiques de symétrie 12 ainsi que sur plusieurs pavages périodiques conduisant aux structures des phases périodiques mentionnées ci-dessus. Le contraste simulé est en excellent accord avec les images expérimentales de microscopie électronique aussi bien pour les structures périodiques que quasi-périodiques. Il reflète essentiellement la structure du pavage sous-jacent aussi pour les structures quasi-périodiques. On en conclut donc que l'interprétation habituelle des images de structure haute résolution est valable non seulement pour les structures périodiques, mais aussi pour les structures dodécagonales non périodiques et que, par conséquent, le schéma de décoration utilisé décrit correctement la structure atomique du quasi-cristal dodécagonal.
\end{abstract}

\begin{abstract}
Since high-resolution electron micrographs of dodecagonal $\mathrm{Ni}-\mathrm{Cr}$ quasicrystals are similar in contrast to those of several closely related periodic phases, it has been argued that dodecagonal quasicrystals can be described as a decoration of a dodecagonal quasiperiodic tiling with the same structural units as occur in these periodic phases. In order to corroborate this hypothesis, electron microscopic contrast simulations using such model structures are presented. The models considered are based on different quasiperiodic, twelvefold-symmetric tilings as well as on several periodic tilings leading to the structures of the periodic phases mentioned above. The simulated contrast is in excellent agreement with the experimental electron microscopic images, both for the periodic and for the quasiperiodic structures. It essentially reflects the structure of the underlying tiling, also for the quasiperiodic structures. It is therefore concluded that the usual interpretation of high-resolution structure images is valid not only for periodic but also for the nonperiodic dodecagonal structures, and that therefore the decoration scheme used correctly describes the atomic structure of the dodecagonal quasicrystal.
\end{abstract}




\section{Introduction.}

Since the discovery of quasicrystals [1] in 1984, a large number of compositionally and structurally different quasicrystalline phases have been found. Besides quasicrystals with icosahedral symmetry, dodecagonal [2], decagonal [3] and octagonal [4] quasicrystals have also been discovered.

The basic structure of quasicrystals can satisfactorily be described by quasiperiodic tilings. However, it is still difficult to determine the atomic decoration of the geometric units of these tilings. Several methods have recently been applied to solve this problem. For example, for icosahedral quasicrystals the Patterson function has been calculated in $3 \mathrm{~d}$ physical space [5] as well as in $6 \mathrm{~d}$ space $[6,7]$, and from this information the atomic decoration can in principle be obtained [8]. Such a Patterson analysis however requires a good quality X-ray powder diffraction pattern, for which a sufficiently large single phase specimen of good quality is necessary.

Since such specimens are not always available, different methods have been applied as well. One of these is the analysis of high-resolution electron microscope (HREM) images, which can be obtained from a single quasicrystalline grain. Given a conjectural model structure, these images can be simulated and compared to the experimental images; in this way the model can either be rejected or used for further application, depending on the quality of the fit between the calculated and the experimental images.

Several of these simulations have been published for icosahedral quasicrystals (see e.g. [9, 10]); however, the simulated images were very similar for different assumed decorations, so that no discrimination between different decorations could be made in this way. In the case of quasicrystals which are periodic in one direction and quasiperiodic in the plane perpendicular to this direction, i.e. for the dodecagonal, decagonal and octagonal quasicrystals, this method appears much more promising however, at least for images taken with the electron beam normal to the quasiperiodic plane. HREM images are essentially determined by the projected potential of the structure, and since the projection direction is in this case a periodic one, it is easier to draw conclusions about such a structure than for structures aperiodic in all three dimensions, especially if the period length is relatively short. The best candidate for the application of this technique is the dodecagonal quasicrystal which occurs in the systems $\mathrm{Ni}-\mathrm{Cr}$ $[2,11,12]$ and Ni-V-Si [13] and has a period length of only $0.46 \mathrm{~nm}$. Apart from the short period length, the dodecagonal quasicrystal is a promising candidate for yet another reason. It typically occurs associated with other well known periodic alloy phases, such as the $\sigma$-phase, the H-phase, and the A15-type structure. These three closely related periodic phases, whose structure can be described by periodic tilings decorated with square and triangular prisms, show HREM images very similar to the dodecagonal quasicrystal. Therefore it may be conjectured that the latter is composed of a quasiperiodic arrangement of the same basic structural units. This is in fact the proposal made originally by Ishimasa et al. [2] (compare also [14]).

The purpose of this paper is to test the correctness of this proposal by detailed contrast calculations. Electron microscopic contrast simulations for the three periodic structures mentioned above as well as for different quasiperiodic model structures are presented and are compared with each other as well as with experimental images. The quasiperiodic model structures are obtained as decorations of different quasiperiodic tilings with the basic structural units taken from the periodic phases. It will be shown that the simulated contrast, which is in excellent agreement with the experimental images, is basically determined by the local arrangement of the tiles, and that this holds for periodic as well as for nonperiodic structures. Since HREM images actually allow conclusions to be made about the local arrangement of the atoms in periodic structures, we can conclude that this atomic 
interpretation of the structure images is also valid for the dodecagonal quasicrystal structure. This strongly supports the proposal made by Ishimasa et al. for the structure of dodecagonal quasicrystals.

\section{Model structures.}

The dodecagonal quasicrystal structure (also termed crystalloid [2]) has first been observed in $\mathrm{Ni}-\mathrm{Cr}$ small particles made by the gas evaporation technique [2]. The ingot used for the evaporation had a bulk composition of $\mathrm{Cr}_{70} \mathrm{Ni}_{30}$. In the simulations this composition has been assumed to be the approximate composition of both the quasiperiodic structure and the associated periodic structures, and it was assumed that no chemical ordering is present. The periodic phases occurring together with dodecagonal quasicrystals can be described by decorations of the tilings shown in figure 1 . The decoration of these tilings with atoms is

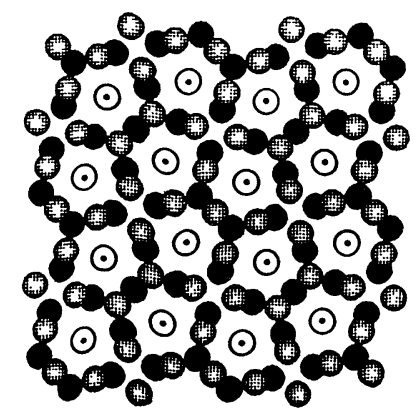

$z=0 \quad 0 z=\frac{1}{2} \quad \odot z= \pm \frac{1}{4}$

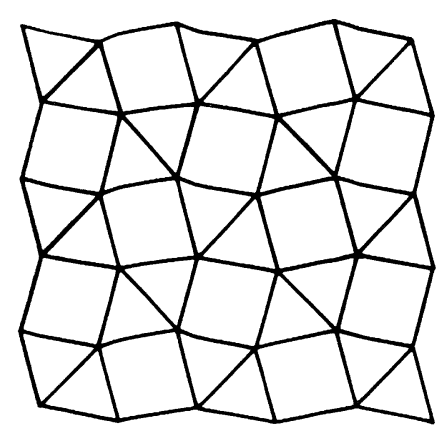

a)
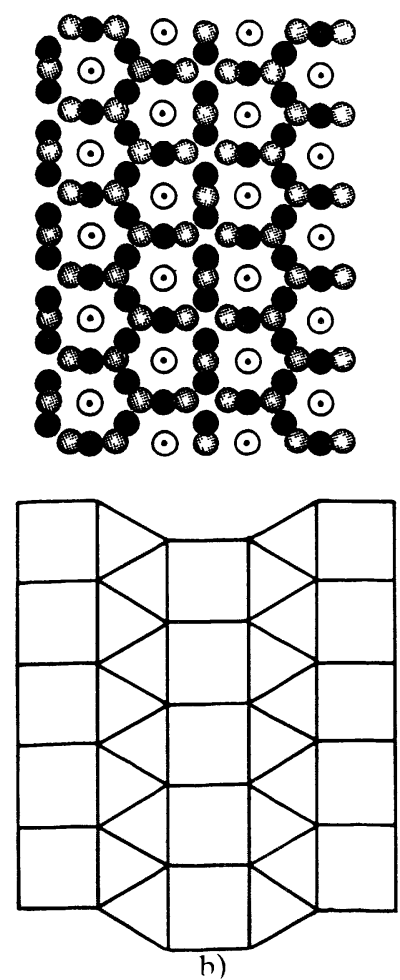
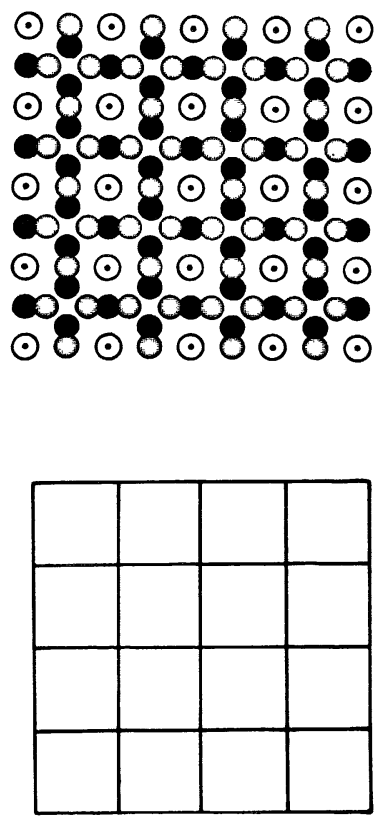

c)

Fig. 1. - Periodic tilings and the corresponding crystal structures related to dodecagonal quasicrystals shown in a $c$-projection. (a) $\sigma$-phase, (b) H-phase and (c) A15-structure.

illustrated in a $c$-projection in figure 1 (top). The $\sigma$-phase and the $\mathrm{H}$-phase structures can be described by periodic arrangements of square and triangular prisms, whereas the A15structure consists of a periodic arrangement of the square prisms only. Note that there are two variants for the decoration of the triangular prisms, depending on the orientation of the triangle. In the H-phase only one variant occurs, whereas in the $\sigma$-phase both are present.

For the image simulation of the dodecagonal quasicrystal, structures based on two different dodecagonal tilings shown in figure 2 were used. The tiling of figure $2 \mathrm{a}$ has been obtained [14, 15] by application of the projection method, and it is therefore truly quasiperiodic. A 


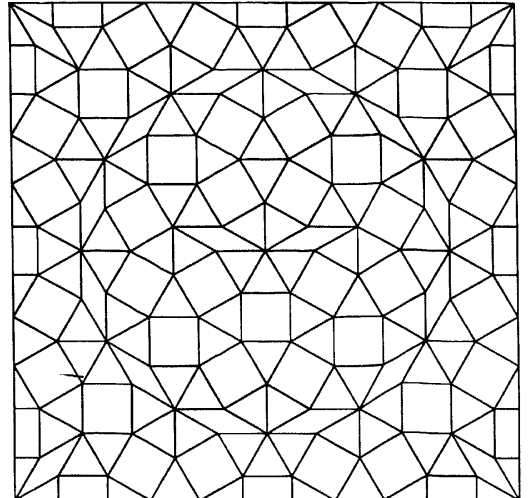

a)

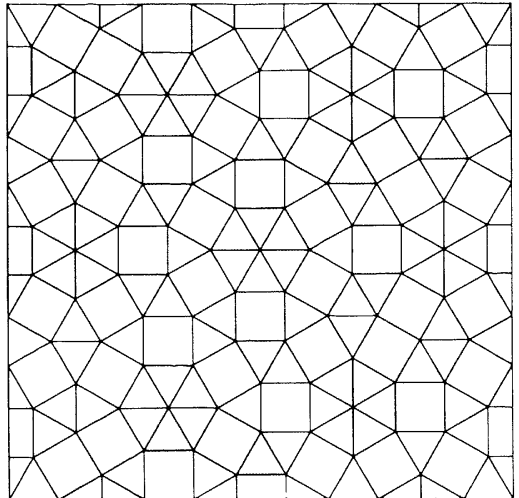

h)

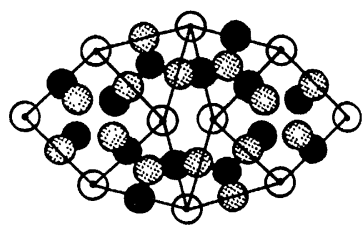

(c)

Fig. 2. - Presentation of two quasiperiodic, dodecagonal tilings with twelvefold Fourier spectrum : (a) truly quasiperiodic tiling, (b) quasiperiodic tiling involving some randomness in the construction (see text), (c) decoration of the basic structural units in the quasicrystal (for an explanation of symbols see Fig. 1).

characteristic feature of this tiling is the occurrence of many rhombic tiles with a $30^{\circ}$ angle. These tiles are interpreted as defects of the quasicrystal structure and do not occur as frequently in the observed images as they do in the model tiling. Note that the same rhombic units also occur as defects in the $\sigma$-phase of Fe-Cr [16]. The squares and triangles of the tiling are again replaced by square and triangular prisms which are decorated in the same way as for the periodic structures discussed above. The decoration of the remaining rhombic prisms is then completely enforced by that of the surrounding ones. The decoration of these basic units is shown in figure $2 \mathrm{c}$. The structure obtained in this way has been analysed in detail in a previous paper [14]. It has the non-symmorphic space group $12_{6} / \mathrm{mmc}$ which contains a screw axis and a set of glide mirror planes.

Figure $2 \mathrm{~b}$ shows a dodecagonal tiling consisting of squares and triangles only, which corresponds more closely to the real structure than the tiling containing many rhombic tiles. It has been generated by an iterated inflation process due to Stampfli [17]. It can be shown [18] that it is essentially quasiperiodic and twelvefold-symmetric, although the arrangement involves a certain amount of randomness. More precisely, in this tiling the orientations of the hexagons inside the dodecagons are selected at random so as to result in a structure with a twelvefold symmetric diffraction pattern. This random choice of the orientation of the hexagons has to be made at all stages of the inflation process. The tiles are again replaced by prisms which are decorated in exactly the same way as for the structures discussed above.

\section{Contrast simulation}

For the contrast simulations of the high-resolution structure images the following electron optical parameters for a JEOL JEM200CX electron microscope have been used : acceleration voltage $200 \mathrm{kV}$, spread of focus $15 \mathrm{~nm}$, spherical aberration constant $C_{\mathrm{s}}=1.2 \mathrm{~mm}$, half angle of convergence $1.2 \mathrm{mrad}$, radius of objective aperture $6.7 \mathrm{~nm}^{-1}$.

All image simulations have been performed with the EMS software package [19]. The multislice technique has been used to simulate the contrast of structure images of the $\sigma$-phase, the $\mathrm{H}$-phase and the A15-type structure as well as the dodecagonal quasicrystal structure. Multislice calculations in a $c$-projection with $128 \times 128$ beams were made for the $\mathrm{Ni}$ - $\mathrm{Cr} \sigma$ - 
phase $(a=0.88 \mathrm{~nm}, c=0.46 \mathrm{~nm})$, with $128 \times 256$ beams for the H-phase $(a=1.717 \mathrm{~nm}$, $b=0.46 \mathrm{~nm}, c=0.46 \mathrm{~nm})$ and with $64 \times 64$ beams for the A15-structure $(a=0.46 \mathrm{~nm})$. The slice thickness corresponded to the length of one $c$-axis parameter of $0.46 \mathrm{~nm}$. For the contrast simulations of the dodecagonal quasicrystal structure, the same slice thickness was used. Since no unit cell can be defined for the nonperiodic quasicrystal structure, the use of an artificial supercell was necessary for multislice calculations. In order to reduce the effect of periodic boundary conditions on the simulated contrast, a rather large supercell dimension was required. The supercells were selected in such a way that periodic boundary conditions were possible without any rearrangement of atoms. This resulted in the following four different sizes of the supercells : $1.72 \mathrm{~nm} \times 1.72 \mathrm{~nm}, 3.43 \mathrm{~nm} \times 3.43 \mathrm{~nm}, 4.69 \mathrm{~nm} \times 4.69 \mathrm{~nm}$ and $6.41 \mathrm{~nm} \times 6.41 \mathrm{~nm}$. For the multislice calculations $256 \times 256$ beams for the smallest supercell and $512 \times 512$ beams for the larger ones were used. Detailed image simulations are presented for one of the larger supercells $(a=4.69 \mathrm{~nm})$ shown in figure 2. For all calculations presented, the absorption was assumed to be zero. However, calculations with realistic absorption were also made and did not show any significant differences in the image contrast. The effect of a smaller slice thickness $(0.23 \mathrm{~nm})$ on the image contrast was investigated for the A15-structure, the $\sigma$-phase as well as for the smallest supercell of the dodecagonal structure. A comparison with the calculations using $0.46 \mathrm{~nm}$ slice thickness did not reveal any differences in the image contrast. It is therefore concluded that the slice thickness chosen is sufficiently small.

Besides the multislice technique the Bloch wave method can also be applied to the image simulation of quasicrystals [10,20]. However, the Bloch wave method is limited to truly quasiperiodic structures. Moreover, if one cannot make use of a high point group symmetry, a very large number of independent beams has to be taken into account in order to obtain reliable results. If such a high point group symmetry is not present, the application of the Bloch wave method results in a prohibitively high demand of computer capacity. Since our second model structure is neither truly quasiperiodic nor exactly twelvefold symmetric, the Bloch wave method cannot be applied. For this reason, all our image simulations have been made using the multislice technique.

All these contrast simulations are then compared to high-resolution structure images obtained from Ni-Cr small particles. These images have been taken by a JEOL JEM200CX high-resolution electron microscope. Since the Ni-Cr small particles change their orientation after two to three exposures, no through focus series could be obtained.

\section{Results.}

For the $\mathrm{Ni}-\mathrm{Cr} \sigma$-phase in the second thickness fringe visible in $200 \mathrm{kV}$ electron micrographs, it was found that the calculated contrast agrees well with the observed one. The second thickness fringe corresponds to $24 \mathrm{~nm}$ to $26 \mathrm{~nm}$, and thicknesses in this range were selected for contrast simulations. Equivalent results using Bloch wave calculations have been obtained by Ishimasa et al. [16a, 21] for the $\mathrm{Fe}-\mathrm{Cr} \sigma$-phase. The contrast of the $\sigma$-phase structure in the $c$-projection consists of four bright spots per unit cell situated at the positions of the atoms with coordinates $z= \pm 1 / 4$; see figure $3 \mathrm{a}$. In figures $3 \mathrm{~b}$-c the contrast calculations for the $\mathrm{H}$ phase and the A15-structure are presented. All calculations in figure 3 correspond to a thickness of $25.3 \mathrm{~nm}$. The images on the left correspond to a defocus value of $59 \mathrm{~nm}$, and the images on the right to the Scherzer defocus value of $67 \mathrm{~nm}$. Figure 4 presents two experimental high-resolution images to $\mathrm{Ni}-\mathrm{Cr}$ small particles containing the $\sigma$-phase, the $\mathrm{H}$ phase and the A15-structure. The contrast simulations show that for thicknesses between $24 \mathrm{~nm}$ and $26 \mathrm{~nm}$ and for defocus values between $55 \mathrm{~nm}$ and $70 \mathrm{~nm}$ (i.e. near optimum 
a)

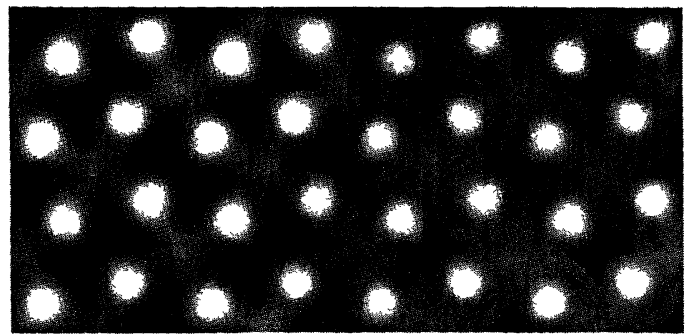

b)

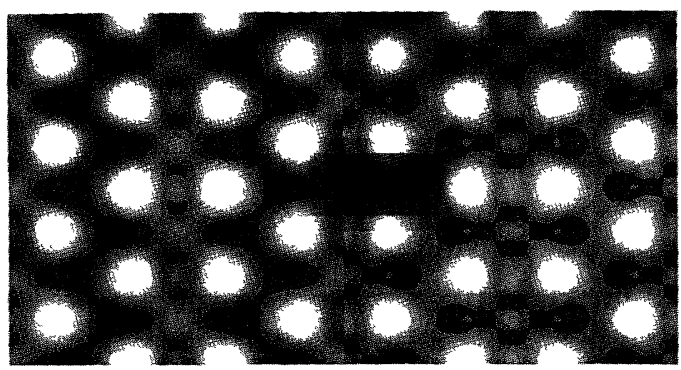

c)

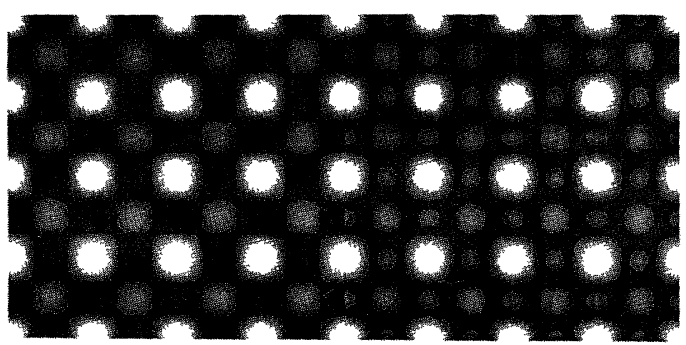

Fig. 3. - Contrast simulations for the $\sigma$-phase (a), the H-phase (b) and the A15-structure for two defocus values : $59 \mathrm{~nm}$ (left) and $67 \mathrm{~nm}$ (right). The thickness corresponds to $25.3 \mathrm{~nm}$ in all calculations.

defocus conditions) the simulated contrast is in excellent agreement with the observed contrast.

The present contrast simulations for the $\sigma$-phase, the $\mathrm{H}$-phase and the A15-structure indicate that in electron microscopic structure images near optimum defocus conditions the bright spots correspond to the vertices of the corresponding tiling composed of squares and triangles.

Contrast simulations for the nonperiodic structure based on the tiling of figure $2 b$ are shown in figure 5 , in which calculations for two different defocus values are presented. On the right hand side of figure 5 the atom positions are superimposed onto these contrast calculations. These calculations correspond to a specimen thickness of $25.3 \mathrm{~nm}$. In figure 6 calculations for the same two defocus values but for a thickness of $27.6 \mathrm{~nm}$ are presented. The figures show that the white dots visible in the contrast calculations correspond to the vertices of the basic tiling.

Contrast simulations for a structure based on the tiling of figure $2 \mathrm{a}$ are presented in figure 7. They correspond to thicknesses of $25.3 \mathrm{~nm}$ and $27.6 \mathrm{~nm}$ and defocus values of $45 \mathrm{~nm}$ and $65 \mathrm{~nm}$. The atom positions are again superimposed onto the calculated contrast. The white dots visible in the simulated contrast again correspond to the vertices of the tiling shown in figure $2 \mathrm{a}$. 
a)

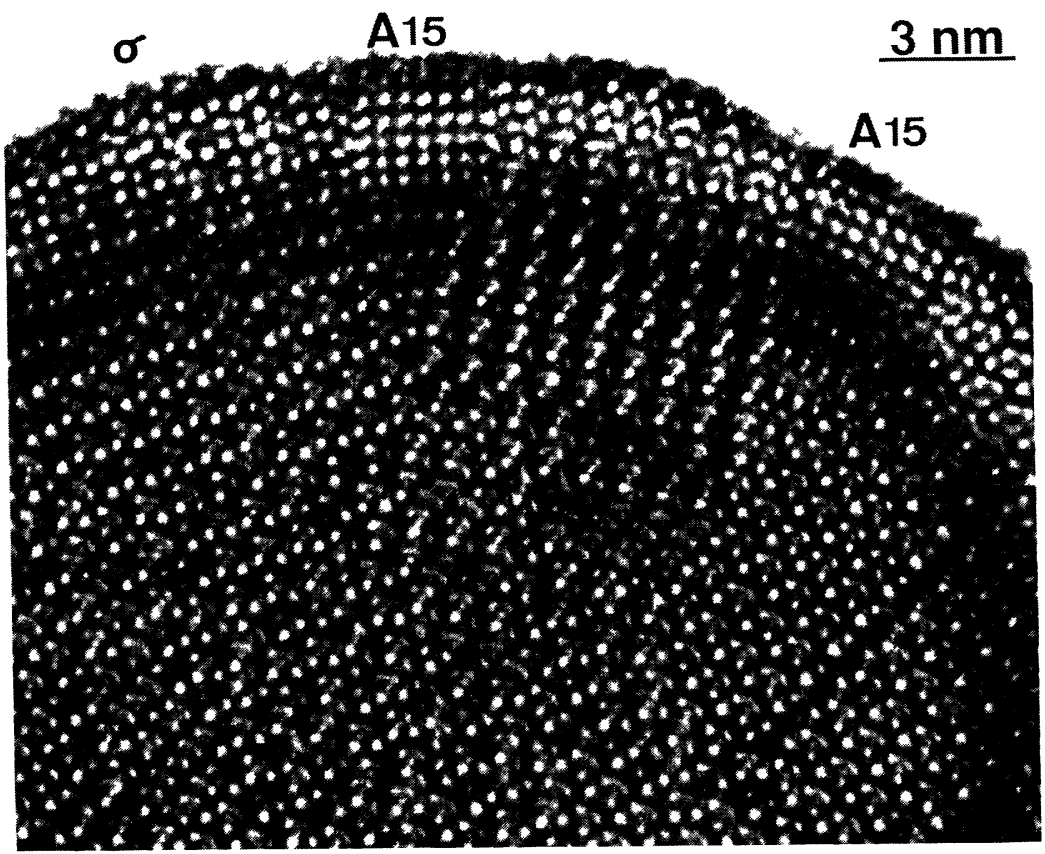

b)

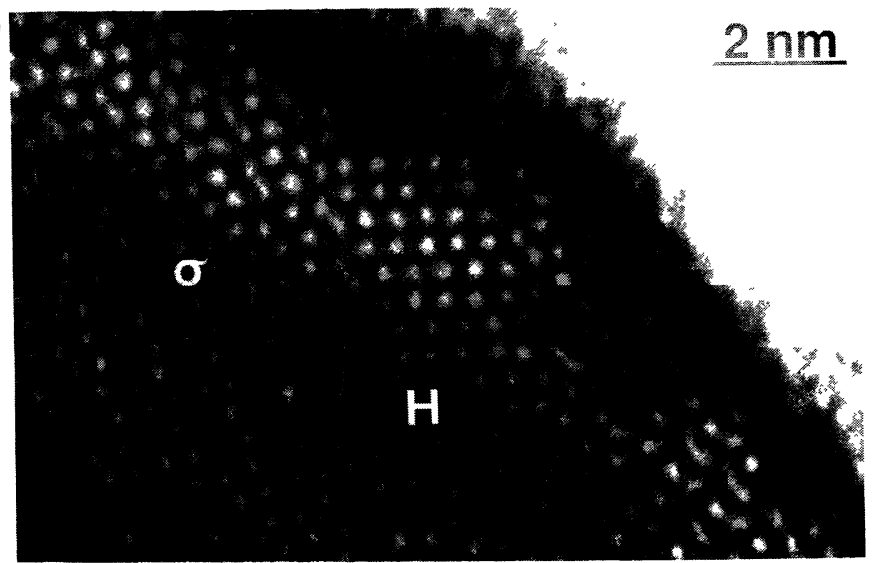

- :

Fig. 4. - Experimental high-resolution images of crystalline Ni-Cr particles. Regions with the $\sigma$-phase, the $\mathrm{H}$-phase and the A15-structure respectively are indicated by symbols. 
a)

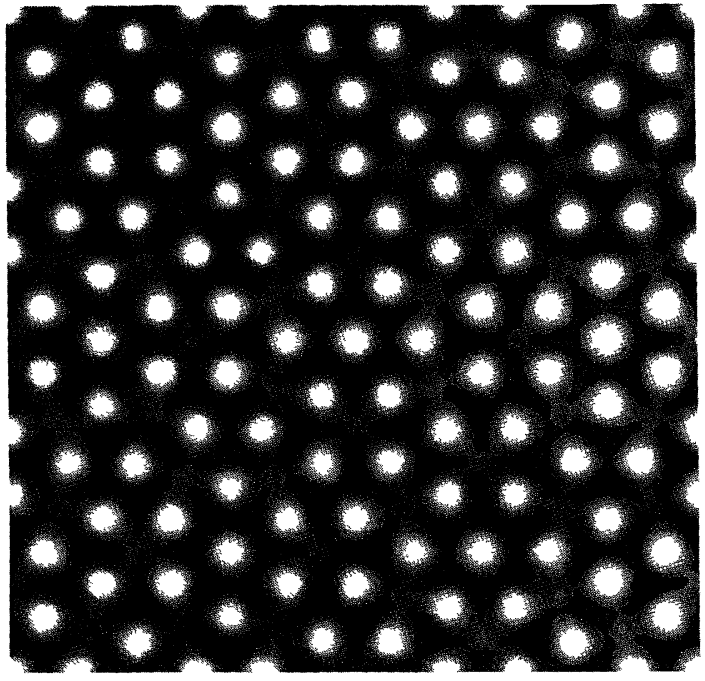

b)

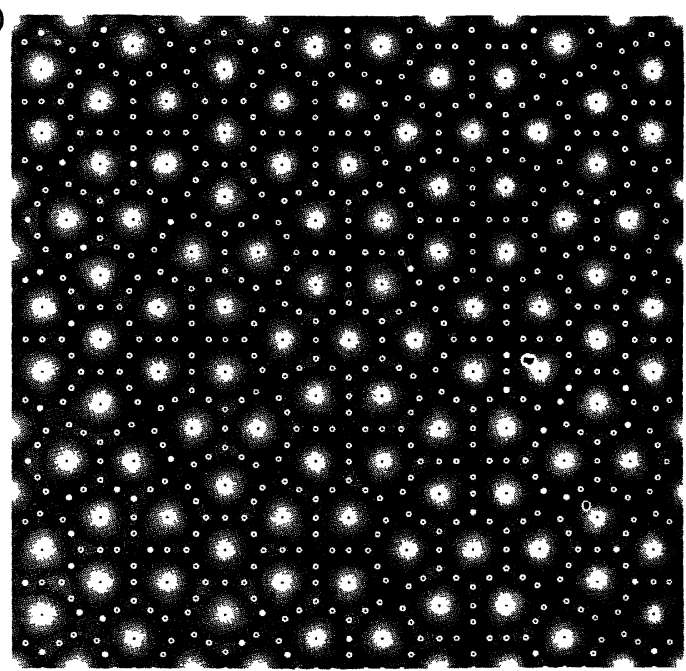

c)

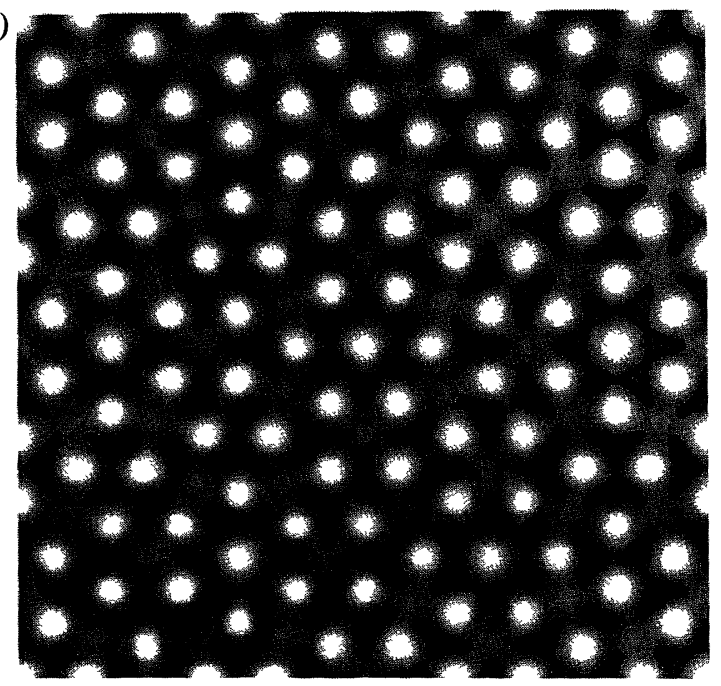

d)

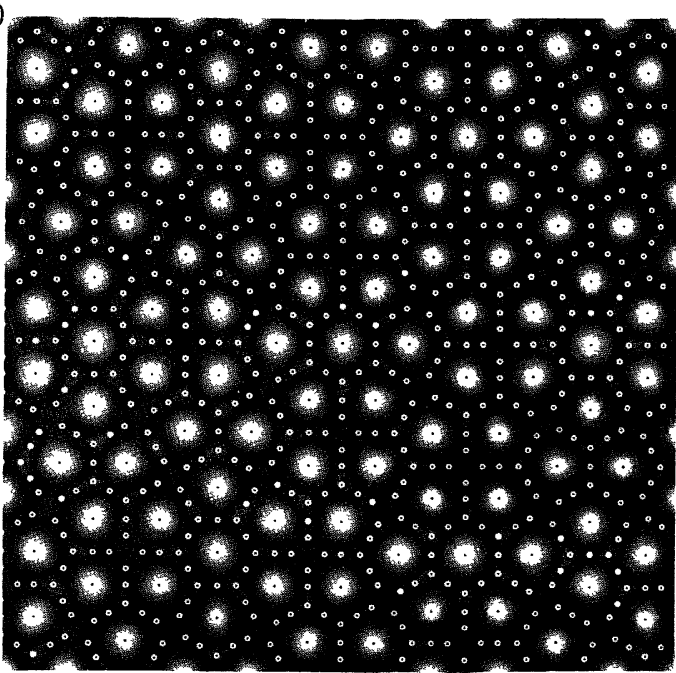

Fig. 5. - Image simulations of the Ni-Cr quasicrystal for a thickness of $25.3 \mathrm{~nm}$ and for two different defocus values : $45 \mathrm{~nm}$ (top) and $55 \mathrm{~nm}$ (bottom). On the right the atom positions are superimposed onto the image. The model structure is based on the tiling shown in figure $2 b$. 


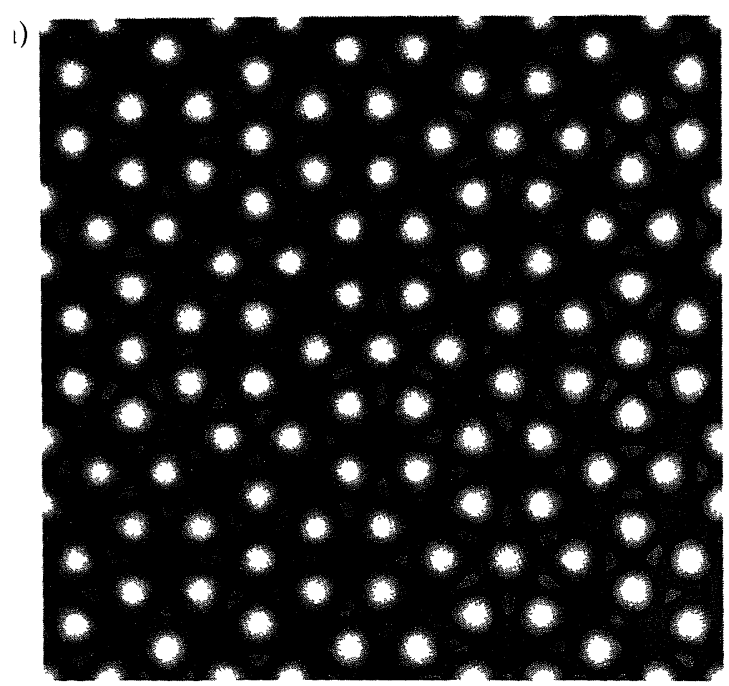

b)
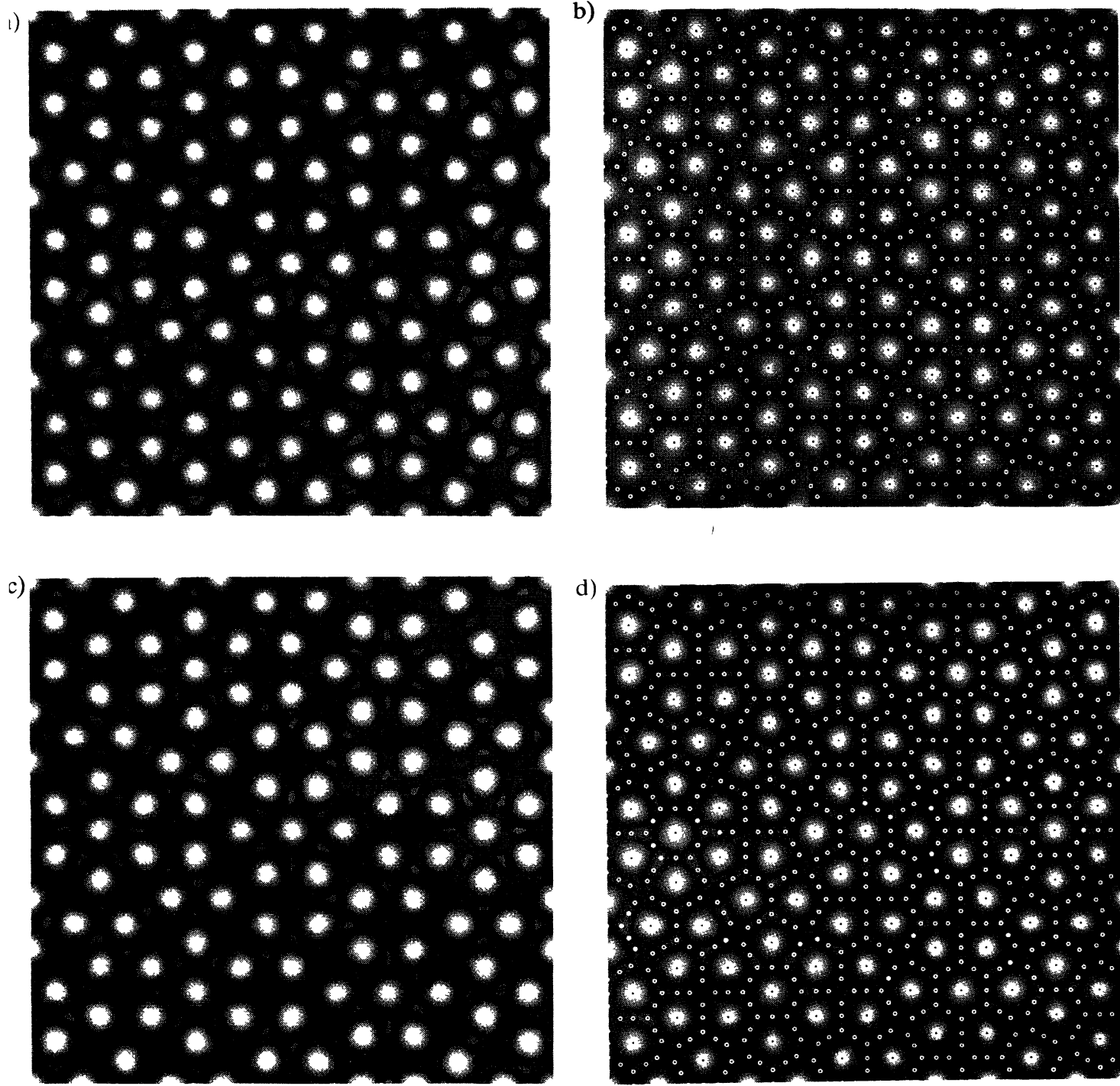

d)

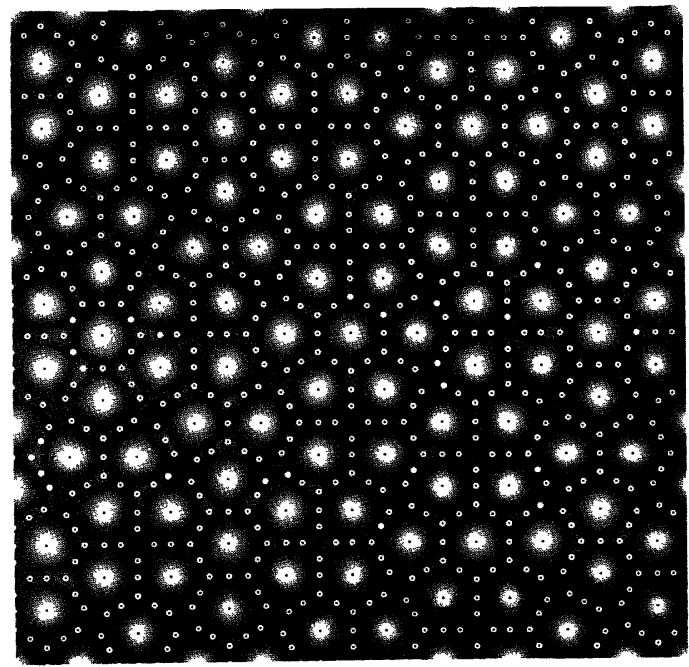

Fig. 6. - Image simulations of the $\mathrm{Ni}-\mathrm{Cr}$ quasicrystal for a thickness of $27.6 \mathrm{~nm}$ and for two different defocus values : $45 \mathrm{~nm}$ (top) and $55 \mathrm{~nm}$ (bottom). On the right the atom positions are superimposed onto the image. The model structure is based on the tiling shown in figure $2 \mathrm{~b}$. 
a)

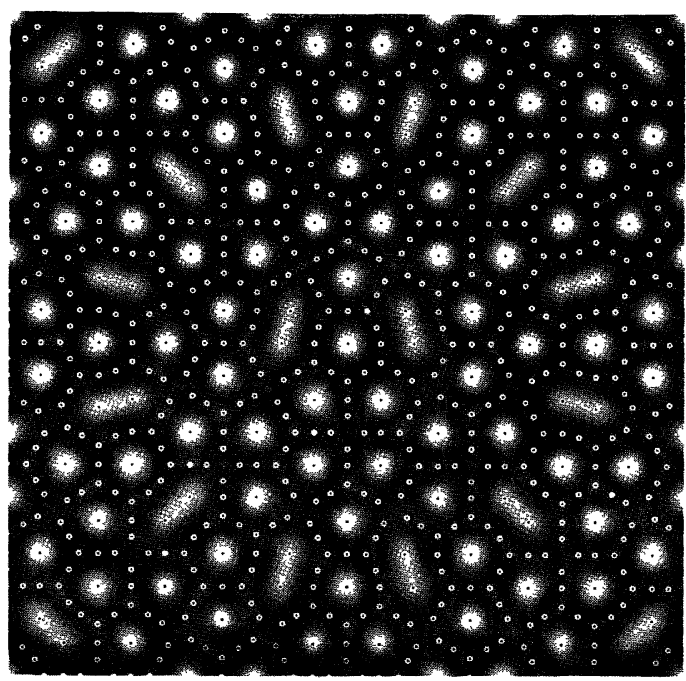

c)

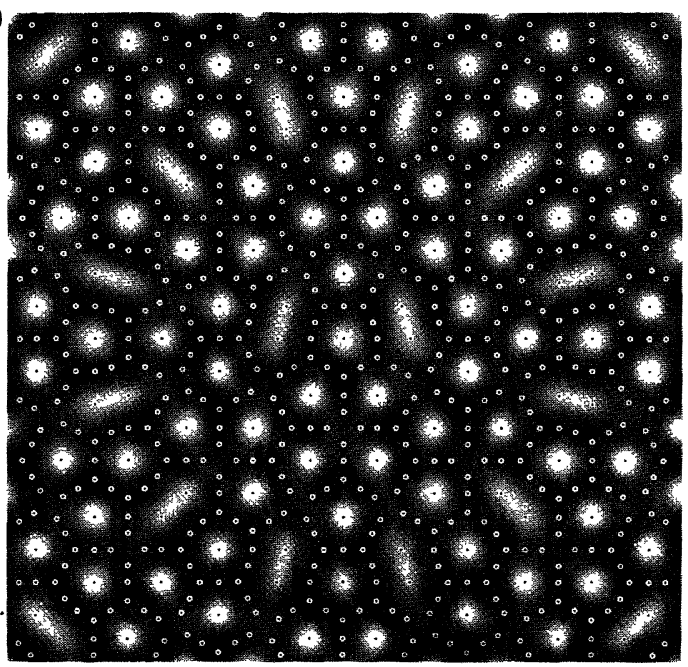

b)

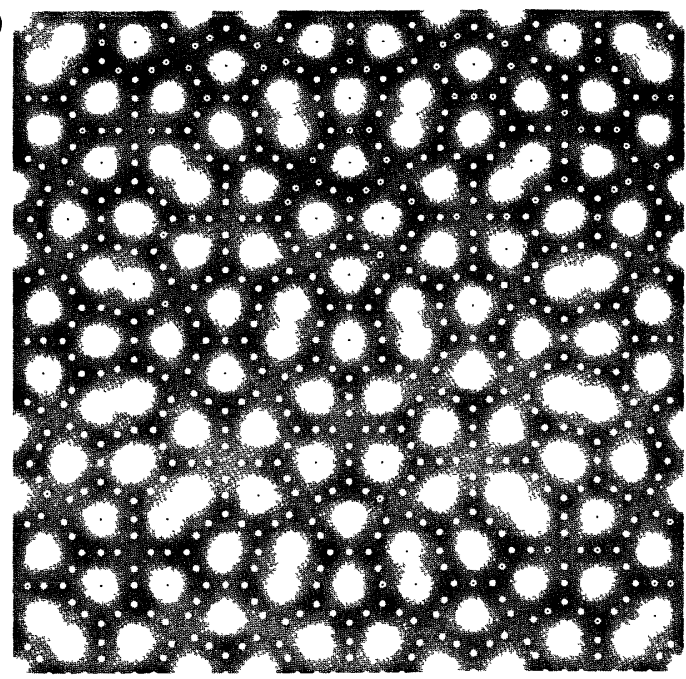

d)

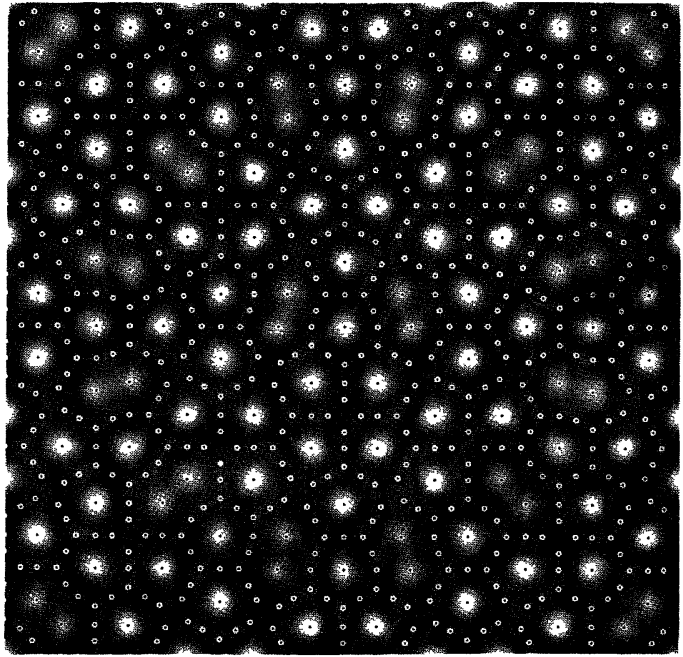

Fig. 7. - Image simulations of the Ni-Cr quasicrystal for two thicknesses : $25.3 \mathrm{~nm}$ (left) and $27.6 \mathrm{~nm}$ (right) and for two different defocus values : $45 \mathrm{~nm}$ (top) and $65 \mathrm{~nm}$ (bottom). The model structure is based on the tiling shown in figure $2 \mathrm{a}$. 
a)

\section{$\underline{2 n m}$}

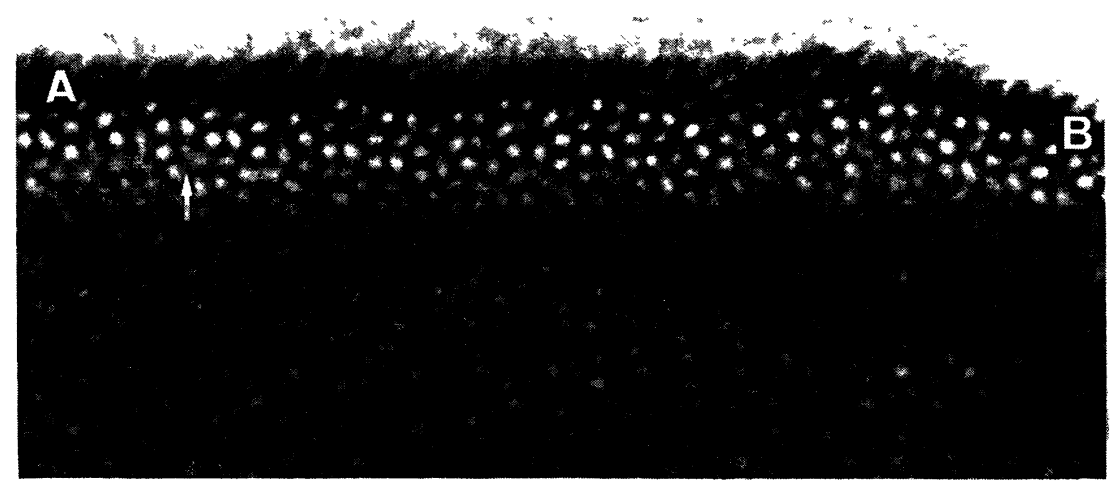

b) $\mathbf{A}$

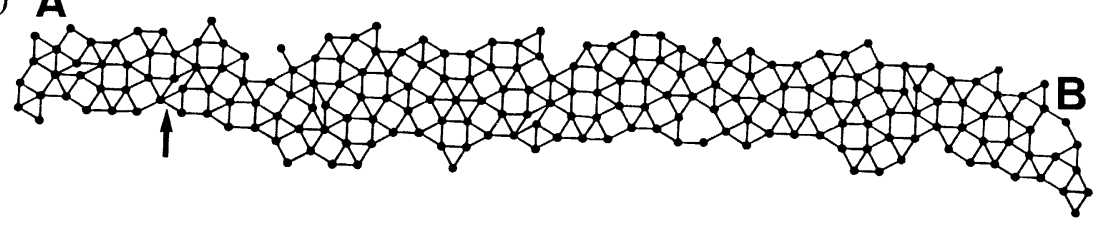

Fig. 8. - (a) Experimental high-resolution image of the dodecagonal $\mathrm{Ni}-\mathrm{Cr}$ quasicrystal. (b) Tiling corresponding to the image shown in (a). Letters A and B indicate how to superimpose the tiling onto the image. The arrow in (a) marks a rhombic unit.

Figure 8a presents a high-resolution image of a $\mathrm{Ni}-\mathrm{Cr}$ quasicrystal showing the typical contrast in the second thickness fringe. In figure $8 \mathrm{~b}$ the tiling corresponding to figure $8 \mathrm{a}$ is shown. The points $\mathrm{A}$ and $\mathrm{B}$ marked in both figures indicate how to superimpose the tiling onto figure $8 \mathrm{a}$. The simulated contrast of the structural units agrees well with the contrast in the observed image, figure $8 \mathrm{a}$. This is true for both dodecagonal model structures. The best correspondence is obtained for a defocus value of $60 \mathrm{~nm}$ and a thickness of approximately $26 \mathrm{~nm} \pm 2 \mathrm{~nm}$. Note that the calculated contrast of the rhombic prisms in excellent agreement with the observations too. One such rhombic unit is marked by an arrow in figure 8 .

The image contrast essentially consists of bright spots situated at the vertex positions of the underlying tiling. This is true for all the periodic as well as the quasiperiodic structures considered here. Therefore the image can be understood as being composed of pieces corresponding to the tiles of the underlying tiling. Pieces which correspond to tiles of the same shape have identical atom decorations. These units in the image contrast thus represent the basic units of the model structures.

It is not surprising that the bright dots are located at the vertices of the underlying tiling. Van Dyck et al. have shown for various alloy systems with a column structure that the vertices of the underlying tiling appear as bright dots [22].

For the periodic structures as well as for the supercells representing parts of the nonperiodic structures, the dependence of the amplitude and the relative phase of the transmitted (000)beam on the specimen thickness has been determined. This relation is identical for all the cases considered here. In particular no difference was found between the periodic and the quasiperiodic structures. 

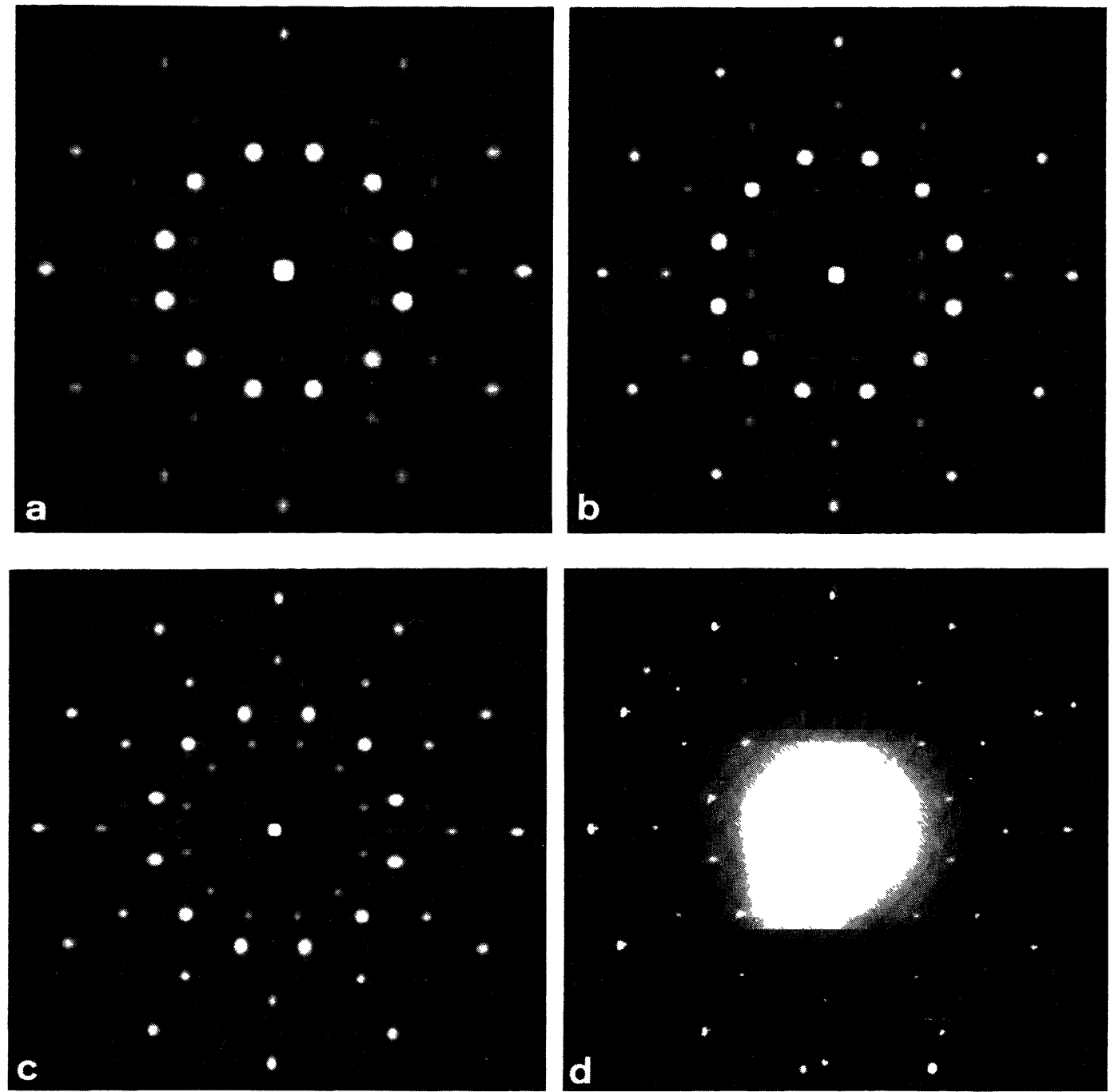

Fig. 9. - Simulated diffraction patterns for supercells of size $a=1.72 \mathrm{~nm}$ (a), $a=4.69 \mathrm{~nm}$ (b) and $a=6.41 \mathrm{~nm}$ (c) compared to the experimental selected area electron diffraction pattern (d).

In addition to the contrast simulations, the corresponding dynamically calculated diffraction patterns have also been obtained. The diffraction patterns of supercells of three different sizes $(a=1.72 \mathrm{~nm}, 4.69 \mathrm{~nm}$ and $6.41 \mathrm{~nm})$, all taken from the exactly quasiperiodic model structure, are shown in figures 9a-c. A comparison with the experimental image, figure 9d, shows an improvement in the quality of the fit with increasing size of the supercell. The calculated diffraction pattern for the smallest supercell still shows a square arrangement of the reflections due the underlying tetragonal supercell, whereas for the larger supercells the deviations from a twelvefold symmetric arrangement of the maxima are smaller. Especially the positions of the weaker reflections show smaller deviations for the larger supercells. Also the intensity distribution shows an improvement with increasing supercell size. Our results imply that supercells considerably larger than $2-3 \mathrm{~nm}$ have to be used in order to obtain an acceptable fit with the experimental diffraction pattern. 


\section{Discussion.}

In the present study the electron microscopic image contrast for two different model structures of the dodecagonal Ni-Cr quasicrystal has been calculated. One of them was based on a truly quasiperiodic dodecagonal tiling, while the construction of the other one involved some randomness, as described above. The decoration of the different tiles was the same as for the $\sigma$-phase, the $\mathrm{H}$-phase and the A15-structure. Since nothing is known about the chemical ordering in the quasicrystal, it was assumed in the calculations presented here that on each atomic position there are $0.7 \mathrm{Cr}$ atoms and $0.3 \mathrm{Ni}$ atoms. Calculations with a partially ordered distribution of the two atomic species were also made. In the ordering scheme used for this calculation it was assumed that the positions with $z$-coordinates $z= \pm 1 / 4$ are occupied by $\mathrm{Cr}$ only. This distribution is analogous to that of the $\mathrm{Ni}-\mathrm{Fe}-\mathrm{Cr} \sigma$-phase. The results showed no detectable differences in the contrast from the calculations assuming total disorder.

It is of interest to compare the thickness and defocus ranges at which the best fit is obtained for the electron microscopic contrast of the crystalline structures and of the quasicrystal structure. The best fit of the calculated contrast to that observed in the $200 \mathrm{kV}$ electron microscope was found for a thickness of $25 \mathrm{~nm} \pm 2 \mathrm{~nm}$ and a defocus value of $60 \mathrm{~nm} \pm 10 \mathrm{~nm}$ for the crystalline structures, whereas for the quasicrystal structure the corresponding values are $26 \mathrm{~nm} \pm 2 \mathrm{~nm}$ (thickness) and $55 \mathrm{~nm} \pm 10 \mathrm{~nm}$ (defocus). It can therefore be concluded that the best fit for crystalline and quasiperiodic structures is obtained at approximately the same thickness and defocus ranges.

A comparison of the contrast simulations for the quasicrystal structures with those for the crystalline structures showed that the contrast of the structural units in the quasicrystals is the same as in the periodic structures. This means that the contrast of the structural units does not depend on their specific local arrangement. Our results therefore indicate that the structural units show the same contrast features even if they are arranged according to a random tiling [23]. This result is of great importance since there are indications [12] that the structure of the dodecagonal quasicrystal is best described by a random tiling.

From the present contrast simulations we conclude that the usual interpretation of highresolution structure images near optimum defocus conditions is valid also for two-dimensional quasiperiodic structures. Therefore the decoration scheme presented here correctly describes the structure of the dodecagonal quasicrystal. In spite of the general lack of experimental through focus series we have thus been able to present evidence that the decoration of the structural units proposed in this paper is correct.

It has been argued [24] that the electron diffraction pattern of dodecagonal quasicrystals could be approximated by that of a rather small periodic approximant with a hexagonal cell of edge length approximately $1 \mathrm{~nm}$. This argument is based exclusively on the kinematic approximation of diffraction. It is well known, however, that in the simulation of electron diffraction patterns of metallic structures, in particular for specimens with the relatively large thicknesses considered here, dynamic scattering effects have to be included in order to produce realistic results. Such dynamic calculations of the electron diffraction patterns for dodecagonal quasicrystals (using the realistic decoration scheme presented here) can be found in reference [14]. It has been found that good agreement with the experimental images could be obtained only if dynamic effects are actually included, while a purely kinematical calculation gives only very poor agreement with experiments. Similar reservations have also been expressed by Kuo [25].

Moreover, as shown in this paper, HREM images of dodecagonal quasicrystals are a very important source of information which should not be neglected. In fact, these HREM images are incompatible with any periodic approximant having a unit cell not larger than a few 
nanometers only. Therefore, a structure such as the model structure proposed by Ho and Li can be excluded for the observed $\mathrm{Ni}-\mathrm{Cr}$ and $\mathrm{Ni}-\mathrm{V}-\mathrm{Si}$ dodecagonal quasicrystals.

\section{Acknowledgments.}

We are very obliged to Dr. T. Ishimasa for stimulating discussions, as well as for making available the diffraction pattern and the high-resolution electron micrographs of $\mathrm{Ni}-\mathrm{Cr}$.

\section{References}

[1] Shechtman D., Blech I., Gratias D. and Cahn J. W., Phys. Rev. Lett. 53 (1984) 1951-1953.

[2] Ishimasa T., Nissen H.-U. and Fukano Y., Phys. Rev. Lett. 55 (1985) 511-513.

[3] Bendersky L., Phys. Rev. Lett. 55 (1985) 1461-1463.

[4] Wang N., Chen H. and Kuo K. H., Phys. Rev. Lett. 59 (1987) 1010-1013.

[5] Cahn J. W., Gratias D. and Mozer B., Phys. Rev. B 38 (1988) 1638-1642.

[6] Gratias D., Cahn J. W. and Mozer B., Phys. Rev. B 38 (1988) 1643-1646.

[7] GÄHLER F., Patterson Analysis of Face-Centered Icosahedral Al-Cu-Fe Quasicrystals, in preparation.

[8] Cahn J. W., Gratias D. and Mozer B., J. Phys. France 49 (1988) 1225-1233.

[9] Ishimasa T., Nissen H.-U., Phys. Scri. T 13 (1986) 291-296.

[10] Cornier M., Zhang K., Portier R. and Gratias D., J. Phys. Colloq. France 47 (1986) C3-447456.

[11] Ishimasa Y., Fukano Y. and Nissen H.-U., in : Quasicrystalline Materials, Eds. Ch. Janot, J. M. Dubois (World Scientific) 1988, pp. 168-177.

[12] Ishimasa T., Nissen H.-U. and Fukano Y., Philos. Mag. A 58 (1988) 835-863.

[13] Chen H., Li D. X. and Kuo K. H., Phys. Rev. Lett. 60 (1988) 1645-1648.

[14] GÄhler F., in : Quasicrystalline Materials, Eds. Ch. Janot, J. M. Dubois (World Scientific), 272284.

[15] Niızeki K. and Mitani H., J. Phys. A : Math. Gen. 20 (1987) L405-L410.

[16] Ishimasa T., J. Sci. Hiroshima Univ. Ser. A 45 (1981) 29-57;

Ishimasa T., Kitano Y. and Komura Y., Phys. Stat. Sol. 66 (1981) 703-715.

[17] Stampfli P., Helv. Phys. Acta 59 (1986) 1260-1263.

[18] GÄHLER F., Quasicrystal Structures from the Crystallographic Viewpoint, Diss. ETH No. 8414 (1988).

[19] Stadelmann P., Ultramicroscopy 21 (1987) 131-145.

[20] Gratias D., Cornier M. and Portier R., Acta Cryst. A 44 (1988) 789-798.

[21] Ishimasa T., Kitano Y. and Komura Y., J. Solid State Chem. 36 (1981) 74-80.

[22] Van Dyck D., Van Tendeloo G. and Amelinckx S., Ultramicroscopy 10 (1982) 263-280;

Van Tendeloo G., VAn Dyck D. and Amelinckx S., Ultramicroscopy 19 (1986) 235-252.

[23] Henley C. L., J. Phys. A : Math Gen. 21 (1988) 1649-1677.

[24] Ho T. L. and Li Y.-H., Phys. Rev. Lett. 62 (1989) 917-920.

[25] Kuo K. H., Phys. Rev. Lett. 63 (1989) 1441. 